\title{
STRUCTURE FORMATION IN SPECTRALLY CONDENSED TURBULENCE
}

\author{
HUA XIA AND MICHAEL SHATS \\ Research School of Physics and Engineering, The Australian National University \\ Canberra, ACT, 0200, Australia \\ Hua.xia@anu.edu.au
}

\begin{abstract}
Two-dimensional (2D) turbulence supports the inverse energy cascade and the spectral condensate generation, which are studied in laboratory experiments. The generation of the spectral condensation via the inverse energy cascade dramatically reduces the radial transport in 2D flows. In this paper we report experimental results related to the formation of spectral condensates. The dynamics of the structural formation is reposted.
\end{abstract}

Keywords: Two-dimensional turbulence; spectral condensation.

\section{Introduction}

Dual cascades of energy and enstrophy predicted by Kraichnan in $2 \mathrm{D}$ turbulence ${ }^{1}$ lead to the spectral power of $E(k)=C_{k} \varepsilon^{2 / 3} k^{-5 / 3}$ for the wave numbers smaller than the wave number $k_{f}$ of the forcing scale, $k<k_{f}$ (the inverse energy cascade range), and to $E(k)=C_{q} \eta^{2 / 3} k^{-3}$ for the small scales, $k>k_{f}$ (forward enstrophy cascade range). Here $\varepsilon$ and $\eta$ are the dissipation rates of energy and enstrophy correspondingly. These two ranges have been found in numerical simulations of 2D turbulence and have also been confirmed in experiments.

It is often said that $2 \mathrm{D}$ turbulence has tendency to generate coherent structures due to the inverse energy cascade. Though this may be true for decaying turbulence, generally this is incorrect. In forced quasi-2D turbulence coherent structures appear as a result of spectral condensation in the presence of a boundary, as a finite system size effect. When this happens, a structure coherent across the box size develops and dominates the flow. Such coherent condensate is a shear flow which modifies underlying turbulence that feeds it. In this paper, we investigate how this new state, referred to as condensed turbulence, is formed.

\section{Spectral Condensation in 2D Fluids}

A good laboratory model of 2D turbulence can be reproduced experimentally in thin stratified layers of electrolyte by electromagnetically generating large number of quasi$2 \mathrm{D}$ vortices. The experimental setup is described in 2 and it is shown schematically in Fig. 1. Turbulent flow is generated in stratified thin layers of electrolyte $\left(\mathrm{Na}_{2} \mathrm{SO}_{4}\right.$ water solution) resting upon heavier non-conducting fluid (Flourinert), each layer is several 
millimeters thick. Turbulence is generated by forcing of up to $900 \mathbf{J} \times \mathbf{B}$-driven vortices in a cell. Spatially varying, vertically directed (normal to the fluid layers) magnetic field $\mathbf{B}$ is produced by a $30 \times 30$ matrix of permanent magnets placed under the bottom of the fluid cell in a checkerboard fashion. Solid Perspex square boundaries of various sizes are inserted to generate spectral condensate. Two platinum electrodes on the either side of the cell are used to run electric current across conducting layer to generate vortices which force turbulence. Flow is visualized using a thin laser sheet $(1 \mathrm{~mm})$ parallel to the free surface. Laser light is scattered by imaging particles suspended in the top layer. Images of the particles are recorded using video camera from above.

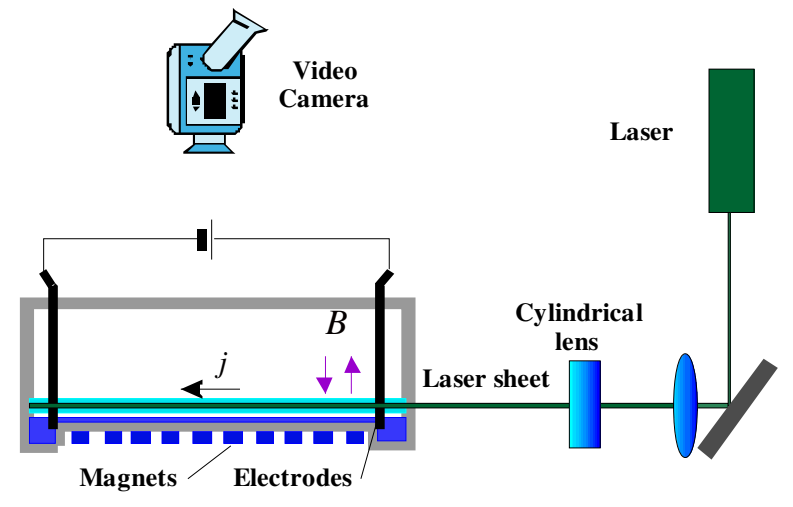

Fig. 1. Schematic of the experimental setup: imaging particles suspended in the top (conducting) layer are illuminated using thin laser sheet. Turbulence is produced by the interacting $\mathbf{J} \times \mathbf{B}$ driven vortices in the top layer. Linear damping is controlled by changing thicknesses of the bottom (non-conducting) layer.

Figure 2 shows particle streaks at 3 different moments of the flow evolution. Shortly after the current is switched on, forcing scale vortices are observed as seen in Fig. 2(a). As the forcing scale vortices $(9 \mathrm{~mm})$ interact with each other, larger eddies are formed due to the transfer of energy from smaller scales (inverse energy transfer), as in Fig. 2(b). If the bottom damping is sufficiently low (this is controlled by changing the thickness of the bottom layer), the size of the largest eddies may become comparable to the boundary size and spectral energy starts accumulating in one big vortex, or spectral condensate, Fig. 2(c). The threshold and conditions of spectral condensation are described in more detail elsewhere ${ }^{3}$.

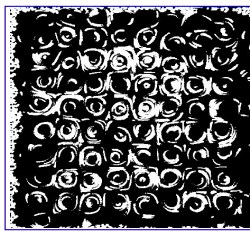

(a)

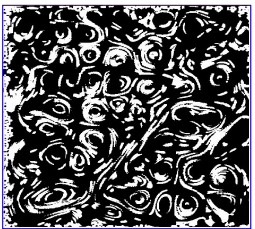

(b)

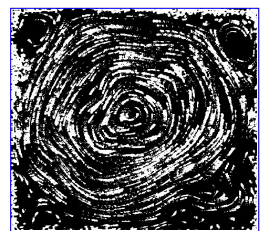

(c)

Fig. 2. Particle streak photos at different stages of the flow evolution on the way to spectral condensation: (a) $t=3 \mathrm{~s}$, (b) $t=11 \mathrm{~s}$, (c) $t=50 \mathrm{~s}$. 


\section{Structure Formation during Spectral Condensation}

Here we focus on the mechanics of the formation of the coherent vortex. First, coherent structures are transiently formed in the flow, even before the generation of a circular vortex. This is illustrated in Fig. 3. Vortices the size of about 1/4 of the box size are formed. The same sign vortices then start destroying the separatrix between them until finally one "circular" vortex forms. The difference between large-scale turbulence eddies, such as those seen in Fig. 2(b) and vortices of Figs. 3(a) and (b) are the lifetime: a lifetime of a vortex is longer than the turnover time. Experimentally we reveal coherent vortices by time-averaging over many instantaneous velocity fields.
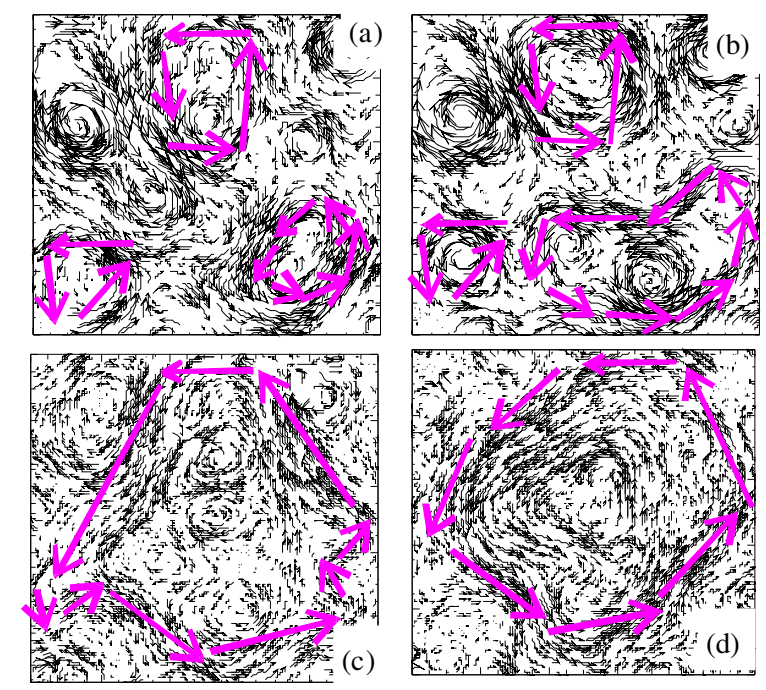

Fig. 3. Time-averaged velocity fields show the merger of the coherent same-sign vortices during the formation of the condensate vortex.

The merger of the vortices into a single circular flow leads to the regularization of turbulence. To qualitatively characterize transport of a passive scalar (which is of interest for comparison with, for example, plasma particle transport) we introduce polar coordinate system in the fluid cell, as shown in Fig. 4(a). The center of it coincides with the center of the box, which is close to the center of the condensate vortex. In this system $2 \mathrm{D}$ velocity can be split into radial $V_{r}$, and azimuthal $V_{\theta}$, components. Then transport of the passive scalar from the center of the box toward the boundary can be characterized by the radial diffusion coefficient

$$
D_{r}=\frac{\left\langle\Delta r^{2}\right\rangle}{\Delta t}=\frac{\Delta t^{2}\left\langle\Delta V_{r}^{2}\right\rangle}{\Delta t}=\Delta t\left\langle\Delta V_{r}^{2}\right\rangle .
$$

Here square brackets denote averaging over the boundary box.

Figure 4(b) shows time evolution of the total kinetic energy of the flow $E_{t}$, and of the radial diffusion coefficient $D_{r}$. For the $D_{r}$ evolution, four dynamical stages can be 
identified. $D_{r}$ grows fast during the stage (1), reaching its maximum and then stays constant during the stage (2). After that, $D_{r}$ decreases rapidly, stage (3), then slowly, stage (4), until it reaches another steady state at $t \approx 65 \mathrm{~s}$. Total kinetic energy $E_{t}=1 / 2 \sum V^{2}$ increases rapidly during the stage (1) together with $D_{r}$, and then slowly grows until reaching its steady-state value during stage (4).

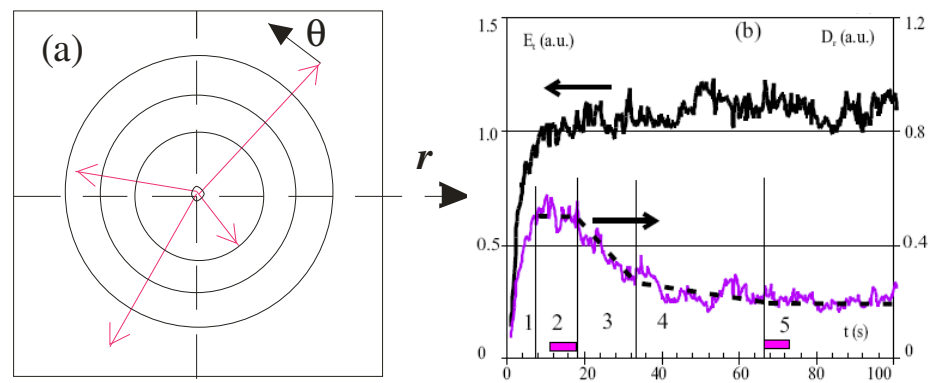

Fig. 4. (a) Polar coordinates with origin in the center of the condensate vortex. (b) Time evolution of the total kinetic energy (top) and of the radial diffusion coefficient (bottom) during spectral condensation of turbulence.

The merger of two co-rotating vortices in 2D fluid has been studied extensively (see e.g. Refs. 4 and 5). It has been shown that an increase in the vortex radius is observed due to the viscous diffusion of vorticity. This induces a simultaneous increase of the ratio between the vortex radius and the separation distance. This quantity also changes in the presence of a third vortex in the neighborhood of the vortex pair. In any case, vortices merge into a bigger vortex in a fast convective process when this aspect ratio reaches critical value. Though during the vortex evolution illustrated above, the vortices are not exactly the same size and the system is constantly forced, we observe qualitatively similar time evolution of the flow on the way to spectral condensation, Fig. 4(b): formation of vortices during stage (1) (Fig. 3(a)), their slow diffusive growth during stage (2) (Fig. 3(b)), then fast convective merger during stage (3) followed by a slow stage (4) (evolution from Figs. 3(c) to (d)) until a symmetry condensate forms. After the condensate vortex is formed, it exists in steady state. The energy is still supplied to it via the inverse energy cascade from the forcing scale ${ }^{6}$.

The four flow development stages identified from the $D_{r}$ evolution shown in Fig. 4 (b) can now be identified as the turbulence development, the condensate, the vortex merger and the symmetrization of the final vortex. These four development stages are observed for several fluid experiments with varying driving current.

\section{Summary}

In thin fluid layers the inverse energy cascade may accumulate at the scale comparable to the system size which leads to spectral condensation and generation of the large-scale vortex. The generation of the spectral condensation reduces the radial diffusion coefficient. 


\section{References}

1. R. H. Kraichnan, Phys. Fluids, 10, 1417 (1967).

2. M. G. Shats, H. Xia, H. Punzmann and G. Falkovich, Phys. Rev. Lett. 99, 164502 (2007).

3. H. Xia, H. Punzmann, G. Falkovich and M. G. Shats, Phys. Rev. Lett. 101, 194504 (2008).

4. P. Meunier, U. Ehrenstein, T. Leweke and M. Rossi, Phys. Fluids, 14, 2757 (2002).

5. C. Cerretelli and C. H. K. Williamson, J. Fluid Mech. 475, 41 (2003).

6. H. Xia, M. Shats and G. Falkovich, Phys. Fluids, 21, 125101 (2009). 